\title{
Eukaryotic Translation Initiation Factor 3 Subunit B Promotes Head And Neck Cancer Via CEBPB Translation
}

\section{Chengzhi Xu}

Fudan University

\section{Yupeng Shen}

Bethune International Peace Hospital

\section{Yong Shi}

Fudan University

Ming Zhang

Fudan University

Liang Zhou ( $\square$ liang.zhou@fdeent.org )

Fudan University https://orcid.org/0000-0003-4344-7278

\section{Research Article}

Keywords: Head and neck cancer, Eukaryotic Translation Initiation Factor 3 Subunit B EIF3B, translation, CEBPB, IL6R,

Posted Date: December 28th, 2021

DOl: https://doi.org/10.21203/rs.3.rs-917405/v1

License: (9) (1) This work is licensed under a Creative Commons Attribution 4.0 International License. Read Full License 


\section{Abstract}

Background: Head and neck squamous cell carcinoma (HNSCC) is the sixth most common cancer type worldwide. Deregulation of mRNA translation is a frequent feature of cancer. Eukaryotic Translation Initiation Factor 3 Subunit B (EIF3B) has reported as oncogenes in cancer. However, the role of EIF3B in HNSCC remains unclear.

Methods: In this study, the clinical significance of EIF3B expression in TCGA was analyzed. Then the expression of EIF3B was knockdown, and its role in HNSC was revealed. To explore the molecular mechanism of EIF3B, we applied RNA sequencing and proteomics, and deregulated pathways were acquired. RNA immunoprecipitation (RIP) sequencing was conducted to uncover the targeting mRNAs of EIF3B. Potential targets of EIF3B were validated with TCGA datasets.

Results: EIF3B serves a hazardous prognostic marker in HNSCC. Besides, EIF3B promotes HNSCC proliferation and progression in vitro and in vivo. EIF3B promotes CEBPB translation and activate MAPK pathway. IL6R and CCNG2 is a target of EIF3B regulated CEBPB translation.

Conclusion: In sum, this study reveals EIF3B as a novel oncogene in HNSCC, by promoting CEBPB translation and IL6R expression.

\section{Highlights}

EIF3B is a risky prognostic biomarker in HNSCC;

EIF3B promotes HNSCC progression in vitro and in vivo;

EIF3B promotes CEBPB translation and activates MAPK pathway;

IL6R and CCNG2 is a target of EIF3B regulated CEBPB translation;

\section{Introduction}

Head and neck squamous cell carcinoma (HNSCC) is the sixth most common cancer type worldwide [1]. HNSCC etiologic factors includes smoking and excessive alcohol consumption, and Human Papilloma Viruses (HPV) infection are risk factors in some HNSCC types [2]. Tumor size, node involvement and smoking habits are some clinical prognosis factors [3]. Due to limited surgical and adjuvant strategies, the 5-year survival of advanced HNSCC patients remains $<50 \%$ [1]. Research of oncogenes in the pathogenesis and development of HNSCC would shed light on discovering novel therapeutic targets, and improve the clinical outcome of patients.

Deregulation of oncogenes and tumor suppressors mRNA translation is a frequent feature of cancer $[4,5]$, and translational regulation has been proposed as potential therapeutic cancer targets [6, 7]. EIF3B (Eukaryotic Translation Initiation Factor 3 Subunit B) is a RNA-binding component of the eukaryotic 
translation initiation factor 3 (elF-3) complexes, which is required for several steps in the initiation of protein synthesis $[8,9]$. The elF-3 complex specifically targets and initiates translation of a subset of mRNAs involved in cell proliferation, including cell cycling, differentiation and apoptosis [10]. The oncogenic role of EIF3B has been widely reported in gastric cancer [11], esophageal carcinoma [12] and bladder cancer [13]. However, the role of EIF3B in HNSCC remains unclear.

Here, the clinical significance of EIF3B in HNSC was analyzed in TCGA and other public datasets. Then the expression of EIF3B was knockdown and its role in HNSC was investigated, in vivo and in vitro. To explore the molecular mechanism of EIF3B, we applied RNA sequencing and proteomics, and deregulated pathways were achieved. At last, RNA immunoprecipitation (RIP) sequencing was conducted to uncover the targeting mRNAs of EIF3B. This study reveals EIF3B as a novel oncogene in HNSCC, by promoting transcription factor CEBPB translation and subsequent IL6R expression.

\section{Material And Methods}

\section{Cell culture}

Human HNSCC cell lines (Fadu and Cal27) were preserved in our lab. Fadu and Cal27 were cultured in Dulbecco's modified Eagle's medium (DMEM, Gibco, Gaithersburg, USA) supplemented with $10 \%$ fetal bovine serum (FBS; HyClone Laboratories, Logan, USA), $100 \mathrm{U} / \mathrm{ml}$ penicillin and $100 \mu \mathrm{g} / \mathrm{ml}$ streptomycin (complete media). All cell cultures were maintained as a monolayer culture at $37^{\circ} \mathrm{C}$ in a humidified atmosphere containing $5 \% \mathrm{CO} 2$.

\section{Lentivirus construction and infection}

To knock down EIF3B expression in cell lines, a recombinant lentivirus expression vector (pGSIL-shEIF3B) containing a green fluorescent protein (GFP) tag was constructed. To generate lentivirus particles, the recombinant expression plasmid was co-transfected with a packaging plasmid system (psPAX2 and pMD2G) into Fadu and Cal27 cells, and viral particles were collected after $48 \mathrm{~h}$. Fadu and Cal27 cells were infected with shEIF3B lentivirus vector or with a negative control vector (Con) for $24 \mathrm{~h}$. The shEIF3B target sequence was as follows:

shRNA1: 5'-GGAAGCAGATGGAATCGATTC-3';

shRNA2: 5'-GGGAGAGAAATTCAAGCAAAT-3';

shRNA3: 5'-GCAAATTCTTTGCCAGAATGA-3'.

\section{Quantitative real-time PCR}

Total RNA was extracted from Fadu and Cal27 cells using TRIzol® RNA Isolation Reagent (Invitrogen, Carlsbad, CA) according to the manufacturer's instructions. Reverse transcription was performed using the PrimeScriptTM RT reagent kit (Takara, Dalian China). All mRNA levels were normalized to the housekeeping gene GAPDH. The following EIF3B primers were used in this study: 5'- 
GGACCCGACCGACTTGAGA-3' (F) and 5'-TTGACCCGGAATGTGTGCTG-3' (R). The GAPDH primers were as follows: 5'-TGACTTCAACAGCGACACCCA-3' (F), 5'- CACCCTGTTGCTGTAGCCAAA-3'(R). All samples were treated under the same conditions and analyzed by qRT-PCR using SYBR Premix Ex Taq ${ }^{\text {TM }}$ (Takara, Dalian China) according to the manufacturer's protocol.

\section{Cell Counting Kit-8 (CCK-8)}

Fadu and Cal27 cells were seeded into 96-well plates $\left(2 \times 10^{4}\right.$ cells/well) and cultured for $12 \mathrm{~h}$. After washing, Fadu and Cal27 cells were incubated with 10\% CCK-8 (Dojindo Molecular Technologies, Inc., Minato-ku, Tokyo, Japan) and optical density measured using a xMark Microporous Plate Absorption Spectrophotometer (Bio-Rad Laboratories, Inc., Hercules, CA, USA).

\section{Cell apoptosis assay}

The apoptotic rate of Fadu and Cal27 cells was detected using an Annexin V, 633 Apoptosis Detection Kit (Dojindo Molecular Technologies), following the kit instructions. Fadu and Cal27 cells were seeded into 6well plates $\left(5 \times 10^{5}\right.$ cells/well $)$ and cultured for $12 \mathrm{~h}$. Next, Fadu and Cal27 were incubated with Annexin $\mathrm{V}$, followed by propidium iodide (PI) buffer for $15 \mathrm{~min}$ at $25^{\circ} \mathrm{C}$ in a dark room. Subsequently, apoptotic cells were quantified using a NovoCyte 1040 flow cytometer (ACEA Biosciences, Inc., Zhejiang, China).

\section{Transwell assay}

Transwell chambers (MilliporeSigma, Burlington, MA, USA) and BD BioCoat Matrigel Invasion Chambers (Franklin Lake, NJ, USA) were used for cell migration and invasion assays. After incubation, the upper chamber residual cells were removed with cotton-tipped swabs, and the cells that passed through the membrane were fixed with $4 \%$ paraformaldehyde and stained with $0.5 \%$ crystal violet. For each experiment, the number of migrating tumor cells was counted from 5 randomly selected fields. Each experiment was repeated at least 3 times.

\section{Wound healing assay}

The migratory ability was assessed through wound-healing assay. Fadu and Cal27 cells $\left(2 \times 10^{5}\right)$ were seeded into a 6-well plate allowed to reach confluence. Then, uniform wounds were scraped using a 200$\mu l$ pipette tip across the cell monolayer. Cells were rinsed with phosphate-buffered saline and cultured in the medium. Then, the wound closures were observed after 24 hours. The initial gap length ( 0 hour) and the residual gap length (24 hours) after wounding were calculated from photomicrographs using an Olympus fluorescence microscope (Olympus).

\section{Nude mice tumor Xeno-graft assay}

Female 4-week-old female nude mice were obtained from Slaccas Company. $2 \times 10^{6}$ cells of each group, at 12 hours after transfection, were injected subcutaneously into the mice. For the same cell line, the normal control group was implanted into the left posterior flank and the knockdown group was in the right of the same mouse. The size of tumors was measured every week by caliper. 3 weeks later, the mice 
were euthanized with $100 \mu \mathrm{L}$ of a 10:1 mixture of ketamine $(100 \mathrm{mg} / \mathrm{mL})$ and xylazine $(100 \mathrm{mg} / \mathrm{mL})$ by injection into the lateral tail vein, the death time was about 1 minute[14].

\section{Transcriptomic RNA sequencing and analysis}

Total RNA was extracted as described above using the NucleoSpin RNA kit (Macherey Nagel, cat. no. 740955). The RNA integrity was monitored using Bioanalyzer analysis (Agilent, RIN: 9.7-10). About 500 pg of RNA per sample was reverse-transcribed and amplified using a modified SMARTseq2 protocol (Rambow et al. 2018). Prior to generating sequencing libraries using the NexteraXT kit (Illumina, cat. no. FC-131-10), cDNA profiles were monitored using the Bioanalyzer. Sequencing was performed on a Nextseq500 platform (Illumina, SE75bp). Reads were then mapped to the human genome (hg19) using STAR (2.4.1b) and quantified with Subread (1.4.6-p2). The results were uploaded to NCBI, and the bioproject accession number is PRJNA664253.

Differential analyses between EIF3B silenced and WT samples were executed using the DeSeq2 pipeline. Genes with RNA expression fold change $>=2$ or $<0.5$ and $p$-value $<0.05$ was defined as significant differential proteins.

\section{Quantitative proteomics analysis}

The proteomic methods were referring to previous publications. Briefly, proteins in EIF3B silenced Fadu cells and control cells were extracted and applied for trypsin digestion. Then spectral library was generated and used for identification and quantitation. Spectronaut $X$ determined the data extraction and the ideal extraction window dynamically, depending on iRT calibration and gradient stability. FDR cutoff on precursor and protein level was $<1 \%$. Decoy generation was set to apply a random number of amino acid position swamps $(\min =2, \max =$ length/2). Otherwise, all the selected fragment ions passing the filters were used for quantification [15]. Proteins with expression fold change $>=2$ or $<0.5$ and $p$-value $<0.05$ was defined as significant differential proteins. The mass spectrometry proteomics data have been deposited to the ProteomeXchange Consortium (http://proteomecentral.proteomexchange.org) via the iProX partner repository [15] with the dataset identifier PXD021532.

\section{RNA immunoprecipitation (RIP) sequencing}

RIP was performed as described with minor modifications [16]. Briefly, Fadu cells were washed and harvested in ice-cold PBS and lysed in RIP lysis buffer. The lysates were incubated on ice for $10 \mathrm{~min}$ and centrifuged at 14,000 rpm for 15 min to clear cell debris. The supernatants were supplemented with NT2 buffer, EDTA to $15 \mathrm{mM}$, DTT to $1 \mathrm{mM}$, RNaseOUT and VRC, and then immunoprecipitated with antiEIF3B/Flag antibody at $4^{\circ} \mathrm{C}$ overnight. The samples were washed five times with NT2 buffer supplemented with 15 mM EDTA. RNA was eluted with TRIsure (Bioline) according to the manufacturer's instructions. The isolated RNA from RIP was analyzed by RNA sequencing.

\section{Statistical analysis}


All statistical analyses were performed using Graphpad v 8.0 software. Each experiment was performed in triplicates, and the data were shown as the mean $\pm S D$, unless otherwise stated. The Kaplan-Meier method was carried out for survival analysis, and the log-rank test was used to estimate differences in survival. A P value $<0.05$ was considered as statistical significance.

\section{Results}

\section{EIF3B act as unfavorable prognostic marker in HNSCC}

To investigate the expression of EIF3B in HNSCC, we first checked its expression in TCGA HNSCC dataset. As shown in Fig. 1A, elevated expression of EIF3B was observed HNSCC cancer samples, compared with control normal group. Then we analyzed the relations between EIF3B expressions with pathological parameters in HNSCC. As shown in Table 1 and Fig. 1B-1H, the expression of EIF3B was significantly correlated with gender, age, HPV infection, $T$ stage, $N$ stage, perineural invasion and survival status. At last, the prognostic significance of EIF3B expression was analyzed with univariate and multivariate Cox regression analysis (Fig. $1 \mathrm{I}$ and $1 \mathrm{~J}$ ), and shown with Kaplan-Meier plot (Fig. 1K). Patients with higher

EIF3B expression showed significant lower survival rates, suggesting that EIF3B serve as an unfavorable prognostic marker in HNSCC. 
Table 1

Correlation of EIF3B expression with clinical parameters in HNSCC.

\begin{tabular}{|c|c|c|c|c|}
\hline & EIF3B & & & \\
\hline Characteristics & Low & High & $\mathrm{X} 2$ & $p$ value \\
\hline & (No. cases) & (No. cases) & & \\
\hline Age & & & 7.946 & 0.005 \\
\hline$\geq 65$ & 6 & 18 & & \\
\hline$<65$ & 43 & 31 & & \\
\hline Gender & & & 5.288 & 0.021 \\
\hline male & 44 & 35 & & \\
\hline female & 5 & 14 & & \\
\hline Stage & & & 3.199 & 0.074 \\
\hline stage1-2 & 13 & 6 & & \\
\hline stage3-4 & 36 & 43 & & \\
\hline $\mathbf{N}$ & & & 0.742 & 0.389 \\
\hline No & 18 & 14 & & \\
\hline N1-3 & 31 & 35 & & \\
\hline $\mathrm{T}$ & & & 17.193 & 0 \\
\hline T1-2 & 29 & 9 & & \\
\hline T3-4 & 20 & 40 & & \\
\hline HPV & & & 14.154 & 0 \\
\hline negative & 26 & 43 & & \\
\hline positive & 23 & 6 & & \\
\hline Tobacco_smoking_history & & & 0.763 & 0.858 \\
\hline Lifelong Non-smoker & 11 & 8 & & \\
\hline Current smoker & 17 & 19 & & \\
\hline Current reformed smoker for $>15$ years & 6 & 7 & & \\
\hline Current reformed smoker for $\leq 15$ years & 13 & 15 & & \\
\hline Perineural_invasion & & & 7.167 & 0.007 \\
\hline
\end{tabular}




\begin{tabular}{|lllll|}
\hline & EIF3B & & & \\
\hline NO & 23 & 16 & & \\
\hline YES & 12 & 29 & 3.967 & 0.046 \\
\hline Status & & & & \\
\hline live & 39 & 30 & & \\
\hline dead & 10 & 19 & \\
\hline
\end{tabular}

\section{EIF3B promotes HNSCC proliferation and progression in vitro and in vivo}

To explore the functional role of EIF3B in HNSCC, we first applied lentivirus mediated EIF3B knockdown (Fig. 2A) in Fadu and Cal27 cell lines. As shRNA2 shows the overall best performance of silencing efficiency, shRNAs was chosen for subsequent phenotype studies. As shown in Fig. 2B-2C, EIF3B silenced Fadu and Cal27 cells exhibits reduced cell number, compared with control cells, suggesting that EIF3B may contributes to cell growth in HNSCC. Then apoptosis rate in EIF3B knockdown and control cells were analyzed. As shown in Fig. 2D, Annexin V/PI staining assay indicated that EIF3B knockdown induced the apoptosis rate, in both cell lines, especially in Fadu cells. Next, transwell and wound healing assays were conducted to explore the impact of EIF3B silencing on cell invasion and migration. As a result, the EIF3B silenced cells demonstrated decreased invasion capability, as shown in Fig. 2E, in both cell lines. Similar to the transwell assay result, the migration distance of HNSCC cell lines showed wider gap, implying that EIF3B knockdown also inhibit cell migration (Fig. 2F). As Fadu cells showed more pronounced overall tumor behavior compared with Cal27 cells, we choose Fadu cells for subsequent in vivo and mechanism study. At last, we applied nude mice xenograft experiment to explore the function of EIF3B in vivo, using Fadu cells. As shown in Fig. 2G, the tumor volume in EIF3B knockdown group was significantly decreased, supporting the oncogenic role of EIF3B in HNSCC.

\section{Integrated analysis reveals CEBPB as a target of EIF3B translation}

To explore the molecular mechanism of the oncogenic role in HNSCC, first, we applied RNA sequencing and label-free proteomics for EIF3B knockdown and Control Fadu cells. The RNA sequencing screened 976 significantly deregulated mRNAs (Fold change $>=2$ or $<=-2, P$ value $<0.05$ ), shown in Fig. 3A. Meanwhile, the quantitative proteomics identified 425 significantly deregulated proteins of all 5195 proteins (Fold change $>=1.5$ or $<=-1.5, \mathrm{P}$ value $<0 .-05$ ), shown in Fig. 3B. Then genes and significant pathways of deregulated mRNAs and proteins were compared. As shown in Fig. 3C, only 14 genes (LAMB3, MYL9, NDRG1, FOSL1, ADAM8, SPRY4, HMOX1, RAB31, PIR, TGFB1, TEF, FAT2, ABR and GRB7) showed both deregulation in mRNA and protein level. In pathway level, there are 103 and 27 significant $(P<0.05)$ pathways for mRNAs and proteins, respectively. For mRNAs, the top3 most significant pathways were pathways in cancer, proteoglycans in cancer, and transcriptional misregulation in cancer. For proteins, the top3 most significant pathways were Ribosome, RNA transport and Ferroptosis. As shown in Fig. 4C, there are 12 common significant pathways enriched in both levels. Based on enrichment score 
( $\log 10 \mathrm{P}$ value), the 12 significant pathways in both levels were shown in Fig. 3D. In addition to MAPK pathways, a well-recognized pathway in cell proliferation and apoptosis, other cell death items, like ferroptosis and necrosis, were significantly enriched, which explains the function of EIF3B regulating cell proliferation and cell death.

EIF3B is a translation initiation factor, and involved in gene translation by binding to mRNAs. Since EIF3B play an oncogenic role in HNSCC, the abnormal expression of EIF3B may influence target genes' translation and protein expression. So we applied RNA immunoprecipitation and RNA sequencing(RIPSeq) to identify potential EIF3B binding mRNAs. 354 mRNAs were identified. Then we compared the 354 mRNAs with 425 significantly deregulated proteins in EIF3B silencing group, and identified 8 genes, VIM, TEF, CALB2, MVD, ASS1, GDF15, NDRG1 and CEBPB (Fig. 4A), and their expression was shown in Fig. 4B. Then we look into studies about the oncogenic and tumor suppressing role of the 8 genes in HNSCC, and finally choose CEBPB as a target candidate. CEBPB is reported to confer radiation resistance of nasopharyngeal carcinoma [17]. As a transcription factor, CEBPB was reported to be translational regulated by EIF6 [18], and regulates MAPK pathway in cancer [19]. To validate the role of CEBPB in mRNA level, we applied ENCODE and ChEA Consensus Transcription factors prediction, and CEBPB was one of the most significant proteins (Fig. 4C). At last, the binding peaks of EIF3B with CEBPB mRNAs and conserved motif were shown in Fig. 4D. After EIF3B was silenced, the binding peaks were reduced, compared with control group. In sum, we identified CEBPB as a translational target of EIF3B, by which EIF3B regulates MAPK and other pathways, in HNSCC.

\section{Transcriptional Targets Of Eif3b Regulated Cebpb Translation}

At last, we explored potential targets of EIF3B regulated CEBPB translation. Fifteen genes were identified by ENCODE and ChEA Consensus Transcription factors prediction, and their expression in EIF3B knockdown and control groups were shown in Fig. 5A. Then we used qRT-PCR to validate 11 genes in EIF3B knockdown and control Fadu cells. In Fig. 5B, 5 genes (SPRY4, NFKBIA, IL6R, CCNG2 and ARID5B) showed consistent significant differential expression. At last, we calculated the co-expression coefficient of EIF3B with the CEBPB and 5 genes. As shown in Fig. 5C, EIF3B showed significant positive correlation with CEBPB $(R=0.23, P=3.1 e-08)$, IL6R $(R=0.27, P=6.2 e-11)$ and SPYR4 $(R=0.27, P=1.5 e-10)$, while showed significant negative correlation with $C C N G 2(R=-0.16, P=0.00013)$. At last, we propose the molecular mechanism of EIF3B in HNSCC: promotes CEBPB translation and regulating targets, like IL6R and CCNG2 (Fig. 5D).

\section{Discussion}

Deregulated translation is characterized as one of the abnormal molecular cancer events, and related genes are emerging as potential therapeutic targets [6]. Studies of family members in Eukaryotic Translation Initiation Factors in HNSCC progression and prognosis are growing. EIF4E is one of the most 
reported genes, and has been reported to cooperates with c-Myc and promotes HNSCC progression [20]. In mammals, the eukaryotic translation initiation factor 3 (elF-3) complex is comprised of 13 subunits: EIF3A-M. The role of EIF3B as a poor prognostic biomarker has been reported in gastric cancer [21], lung cancer [21, 22], glioma [23], ovarian cancer[24] and renal cancer [25]. Meanwhile, EIF3B were also an oncogene in gastric cancer [11], lung cancer [21,22, 26], osteosarcoma[27], esophageal squamous cell carcinoma [12, 28], leukemia [29] and renal cancer [25]. In HNSCC, the elF-3 complex is recruited by DDX3, and promote HNSCC metastasis, by translating upstream open reading frames (UORF) containing oncogenic mRNAs [30]. The role of EIF3B in HNSCC was unclear.

In this study, we analyzed the clinical significance of EIF3B in HNSCC. Consistent with other cancer types mentioned above, EIF3B showed significant higher expression in HNSCC, and patients with elevated expression showed worse prognosis, indicating a hazardous role of EIF3B in HNSCC prognosis.

Interestingly, we also found that HPV positive HNSCC patients showed significant lower EIF3B expression, but whether the HPV infection influences EIF3B expression remains to be explored. Another founding is the significant correlation of EIF3B expression with perineural invasion in HNSCC. Perineural invasion (PNI) is a mechanism of tumor dissemination via nerves, and is associated with poor clinical outcomes [31]. By now, PNI markers, like neurotropic factors NGF and BDNF, have been proposed [32]. The significance of EIF family member elF-4E with perineural invasion was reported in colorectal cancer [33], and the correlation of EIF3B in HNSCC was first reported in this study. The significant co-expression of EIF3B with PNI markers also supported the conclusion of EIF3B as PNI marker. In the future, we would explore the role of EIF3B in HNSCC perineural invasion.

CEBPB is a transcription factor widely reported in cancer. In nasopharyngeal carcinoma and HNSCC, CEBPB was involved in PGC1 a mediated radiation resistance [17, 34]. The abnormal translational of CEBPB [35] has been widely reported in macrophage migration[36], metabolic diseases [37] and leukemia [38]. EIF6 was previously described as a CEBPB translational regulator, mainly in adipogenic regulation [18]. We identified CEBPB as a translation target of EIF3B, and proposed its oncogenic role by modulating downstream targets, like IL6R and CCNG2.

\section{Conclusion}

In this study, the function of EIF3B in HNSCC was studied, and lentivirus mediated EIF3B knockdown inhibited the proliferation, migration and invasion capability, in vivo and in vitro. These results indicated EIF3B as an oncogene in HNSCC. Meantime, there are some limits in this part. First, this study silenced the expression of EIF3B in Fadu and Cal27 cell lines, the effect of EIF3B overexpression can also been applied. Secondly, we proposed that EIF3B promotes HNSCC via CEBPB translation, and rescue assay of whether CEBPB protein up-regulation would phenocopy the function of EIF3B would provide more solid evidence for the conclusion. At last, the clinical significance of EIF3B in HNSCC was mainly based on public database of TCGA. If possible, the significance may be validated with more HNSCC specimen and clinical data. 


\section{Declarations}

\section{Ethics approval and consent to participate}

Animal experiments were approved by the Animal Care and Use Committee of Head and Neck Surgery, Eye Ear Nose and Throat Hospital.

\section{Consent for publication}

Informed consent for publication of the manuscript was obtained from all authors.

\section{Availability of data and materials}

All relevant data can be acquired by contacting the corresponding author.

\section{Competing interests}

All authors declared no competing interests.

\section{Funding}

This work was supported by the Natural Science Foundation of Shanghai (16ZR1419300).

\section{Acknowledgements}

This work was supported by the Natural Science Foundation of Shanghai (16ZR1419300).

\section{Authors' contributions}

Chengzhi Xu and Liang Zhou designed the research. Chengzhi Xu, Yupeng Shen, and Yong Shi performed the molecular cell experiments. Chengzhi Xu and Ming Zhang performed bioinformatics analysis. Yupeng Shen carried out visualization. Liang Zhou wrote manuscript. All authors reviewed and approved the final manuscript.

\section{References}

1. Global Burden of Disease. Cancer C, et al., Global, Regional, and National Cancer Incidence, Mortality, Years of Life Lost, Years Lived With Disability, and Disability-Adjusted Life-years for 32 Cancer Groups, 1990 to 2015: A Systematic Analysis for the Global Burden of Disease Study. JAMA Oncol, 2017. 3(4): 524-48.

2. Leemans CR, Snijders PJF, Brakenhoff RH. The molecular landscape of head and neck cancer. Nat Rev Cancer. 2018;18(5):269-82.

3. Kang $\mathrm{H}$, Kiess A, Chung $\mathrm{CH}$. Emerging biomarkers in head and neck cancer in the era of genomics. Nat Rev Clin Oncol. 2015;12(1):11-26.

4. Tahmasebi S, et al. Translation deregulation in human disease. Nat Rev Mol Cell Biol. 2018;19(12):791-807. 
5. Bramham CR, Jensen KB, Proud CG. Tuning Specific Translation in Cancer Metastasis and Synaptic Memory: Control at the MNK-elF4E Axis. Trends Biochem Sci. 2016;41(10):847-58.

6. Blagden SP, Willis AE. The biological and therapeutic relevance of mRNA translation in cancer. Nat Rev Clin Oncol. 2011;8(5):280-91.

7. Bhat $M$, et al. Targeting the translation machinery in cancer. Nat Rev Drug Discov. 2015;14(4):26178.

8. Aylett $\mathrm{CH}$, et al. Structure of a yeast 40S-elF1-elF1A-elF3-elF3j initiation complex. Nat Struct Mol Biol. 2015;22(3):269-71.

9. Valasek L, Nielsen KH, Hinnebusch AG. Direct elF2-elF3 contact in the multifactor complex is important for translation initiation in vivo. EMBO J. 2002;21(21):5886-98.

10. Feng X, Li J, Liu P. The Biological Roles of Translation Initiation Factor 3b. Int J Biol Sci. 2018;14(12):1630-5.

11. Ma F, et al. Downregulation of eukaryotic translation initiation factor $3 \mathrm{~b}$ inhibited proliferation and metastasis of gastric cancer. Cell Death Dis. 2019;10(9):623.

12. Xu F, et al. TEX9 and elF3b functionally synergize to promote the progression of esophageal squamous cell carcinoma. BMC Cancer. 2019;19(1):875.

13. Wang $\mathrm{H}$, et al. Translation initiation factor elF3b expression in human cancer and its role in tumor growth and lung colonization. Clin Cancer Res. 2013;19(11):2850-60.

14. Schoell $A$, et al. Euthanasia method for mice in rapid time-course pulmonary pharmacokinetic studies. Journal of the American Association for Laboratory Animal Science: JAALAS. 2009;48(5):506-11.

15. Zhang Q, et al. ACOX2 is a prognostic marker and impedes the progression of hepatocellular carcinoma via PPARalpha pathway. Cell Death Dis. 2021;12(1):15.

16. Hsu JC, et al. Oncoprotein AEG-1 is an endoplasmic reticulum RNA-binding protein whose interactome is enriched in organelle resident protein-encoding mRNAs. RNA. 2018;24(5):688-703.

17. Du Q, et al. PGC1alpha/CEBPB/CPT1A axis promotes radiation resistance of nasopharyngeal carcinoma through activating fatty acid oxidation. Cancer Sci. 2019;110(6):2050-62.

18. Brina $D$, et al. elF6 coordinates insulin sensitivity and lipid metabolism by coupling translation to transcription. Nat Commun. 2015;6:8261.

19. Xia P, Zhang R, Ge G. C/EBPbeta Mediates TNF-alpha-Induced Cancer Cell Migration by Inducing MMP Expression Dependent on p38 MAPK. J Cell Biochem. 2015;116(12):2766-77.

20. Ruggero $D$, et al. The translation factor elF-4E promotes tumor formation and cooperates with c-Myc in lymphomagenesis. Nat Med. 2004;10(5):484-6.

21. Wang L, et al. EIF3B is associated with poor outcomes in gastric cancer patients and promotes cancer progression via the PI3K/AKT/mTOR signaling pathway. Cancer Manag Res. 2019;11:787791. 
22. Tian Y, et al. EIF3B correlates with advanced disease stages and poor prognosis, and it promotes proliferation and inhibits apoptosis in non-small cell lung cancer. Cancer Biomark. 2018;23(2):291300 .

23. Chai RC, et al. Systematically profiling the expression of elF3 subunits in glioma reveals the expression of elF3i has prognostic value in IDH-mutant lower grade glioma. Cancer Cell Int. 2019;19:155.

24. Zhao N, et al., Eukaryotic initiation factor $3 B$ is overexpressed and correlates with larger tumor size, advanced FIGO stage, and shorter overall survival in epithelial ovarian cancer patients. J Clin Lab Anal, 2020: p. e23354.

25. Zang $\mathrm{Y}$, et al. Eukaryotic Translation Initiation Factor $3 \mathrm{~b}$ is both a Promising Prognostic Biomarker and a Potential Therapeutic Target for Patients with Clear Cell Renal Cell Carcinoma. J Cancer. 2017;8(15):3049-61.

26. Jin $\mathrm{D}$, et al., $m(6) A$ mRNA methylation initiated by METTL3 directly promotes YAP translation and increases YAP activity by regulating the MALAT1-miR-1914-3p-YAP axis to induce NSCLC drug resistance and metastasis. J Hematol Oncol, 2019. 12(1): p. 135.

27. Choi YJ, et al. Silencing of translation initiation factor elF3b promotes apoptosis in osteosarcoma cells. Bone Joint Res. 2017;6(3):186-93.

28. Xu F, et al. Eukaryotic translation initiation factor 3B accelerates the progression of esophageal squamous cell carcinoma by activating beta-catenin signaling pathway. Oncotarget. 2016;7(28):43401-11.

29. Huang L, et al., elF3b regulates the cell proliferation and apoptosis processes in chronic myelogenous leukemia cell lines via regulating the expression of C3G. Biotechnol Lett, 2020.

30. Chen HH, et al. DDX3 Activates CBC-elF3-Mediated Translation of uORF-Containing Oncogenic mRNAs to Promote Metastasis in HNSCC. Cancer Res. 2018;78(16):4512-23.

31. Bakst RL, et al. Perineural Invasion and Perineural Tumor Spread in Head and Neck Cancer. Int J Radiat Oncol Biol Phys. 2019;103(5):1109-24.

32. Schmitd LB, Scanlon CS, D'Silva NJ. Perineural Invasion in Head and Neck Cancer. J Dent Res. 2018;97(7):742-50.

33. Chen YT, et al. Eukaryotic translation initiation factor 4E (elF-4E) expressions are associated with poor prognosis in colorectal adenocarcinoma. Pathol Res Pract. 2017;213(5):490-5.

34. Jerhammar F, et al. Fibronectin 1 is a potential biomarker for radioresistance in head and neck squamous cell carcinoma. Cancer Biol Ther. 2010;10(12):1244-51.

35. Peeper DS. C/EBPbeta: lost beyond translation. EMBO J. 2011;30(18):3663-4.

36. Perino A, et al. TGR5 reduces macrophage migration through mTOR-induced C/EBPbeta differential translation. J Clin Invest. 2014;124(12):5424-36.

37. Zidek LM, et al. Deficiency in mTORC1-controlled C/EBPbeta-mRNA translation improves metabolic health in mice. EMBO Rep. 2015;16(8):1022-36. 
38. Guerzoni $C$, et al. Inducible activation of $C E B P B$, a gene negatively regulated by $B C R / A B L$, inhibits proliferation and promotes differentiation of BCR/ABL-expressing cells. Blood. 2006;107(10):40809.

\section{Figures}

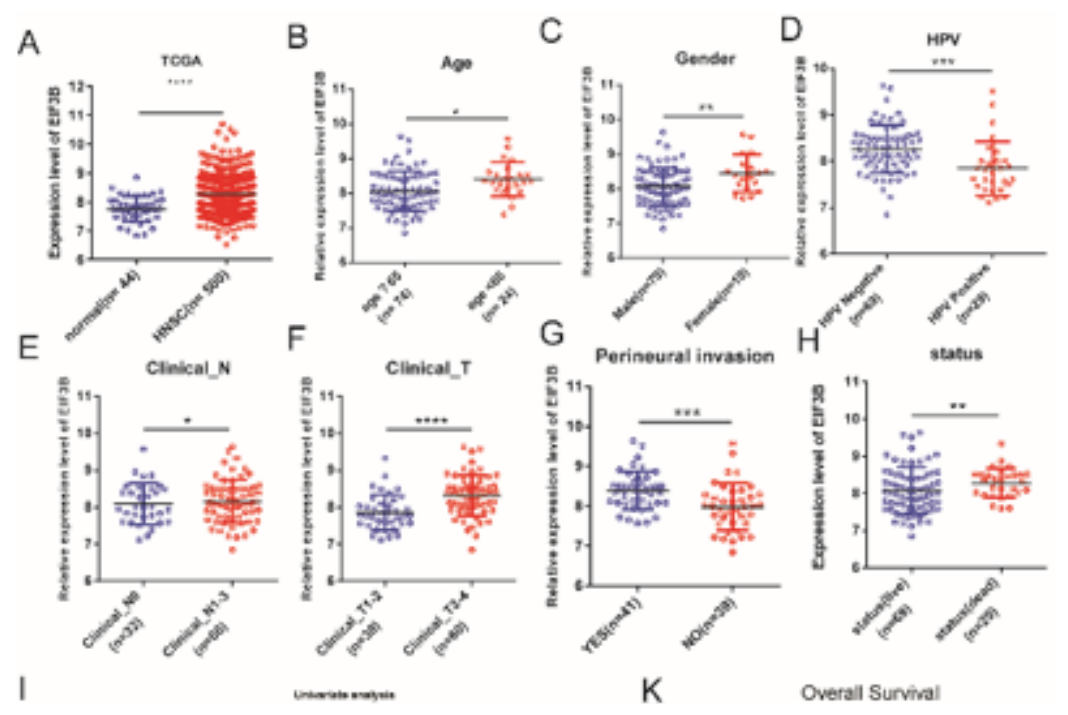

\section{Figure 1}

\section{EIF3B is a risky prognosticator in HNSCC.}

(A) In TCGA HNSC dataset, EIF3B also shows significant higher expression in cancer samples; The expression of EIF3B is significantly correlated with clinical Age (B), Gender (C), HPV infection (D), ClinicalT stage (E), Clinical-N stage (F), Perineural invasion $(G)$ and outcome status $(H)$.

(I) Univariate COX analysis of EIF3B and other clinical parameters with HNSCC survival; (J)Multivariate COX analysis of EIF3B and other clinical parameters with HNSCC survival; (K) Kaplan-Meier plot shows EIF3B as a hazardous prognosticator in HNSCC. *, $\mathrm{P}<0.05$;

Figure 2 
EIF3B knockdown reduces HNSCC in vitro and in vivo.

(A) EIF3B was knockdown using shRNAs, and the knockdown efficiency was examined with qRT-PCR in Fadu and Cal27s; (B-C) CCK8 assay indicated EIF3B knockdown attenuated cell proliferation of Fadu and Cal27 cells;

(D) Cell apoptosis rate was increased in Fadu and Cal27 cells, in ElF3B Knockdown groups; (E) Cell invasion was examined with transwell assay in Fadu and Cal27 cells; (F) Cell migration was examined with wound healing assay in Fadu and Cal27 cells; $(\mathrm{G})$ In vivo nude mice xenograft result shows that EIF3B knockdown reduced the tumor volume of Fadu $(n=5)$.

All assays were replicated three times. ${ }^{*}, \mathrm{P}<0.05 ; * \star, \mathrm{P}<0.01$.

A

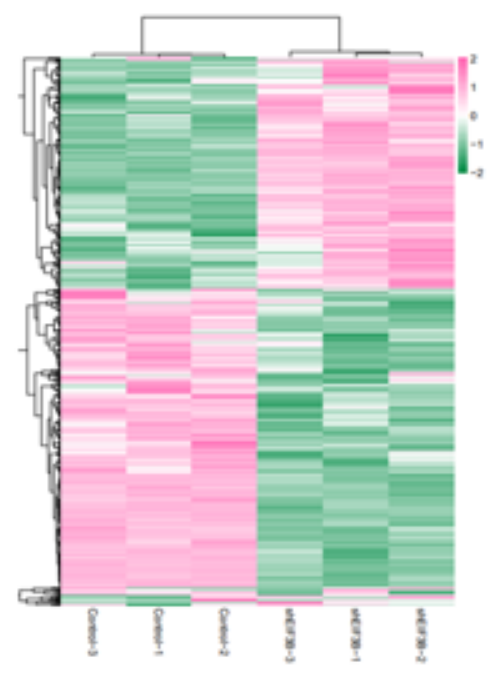

C

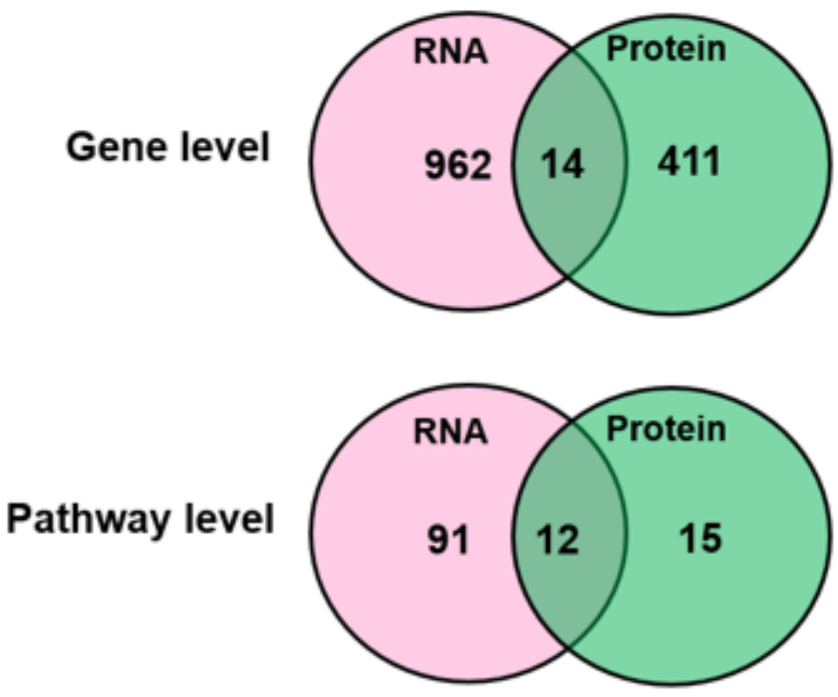

B
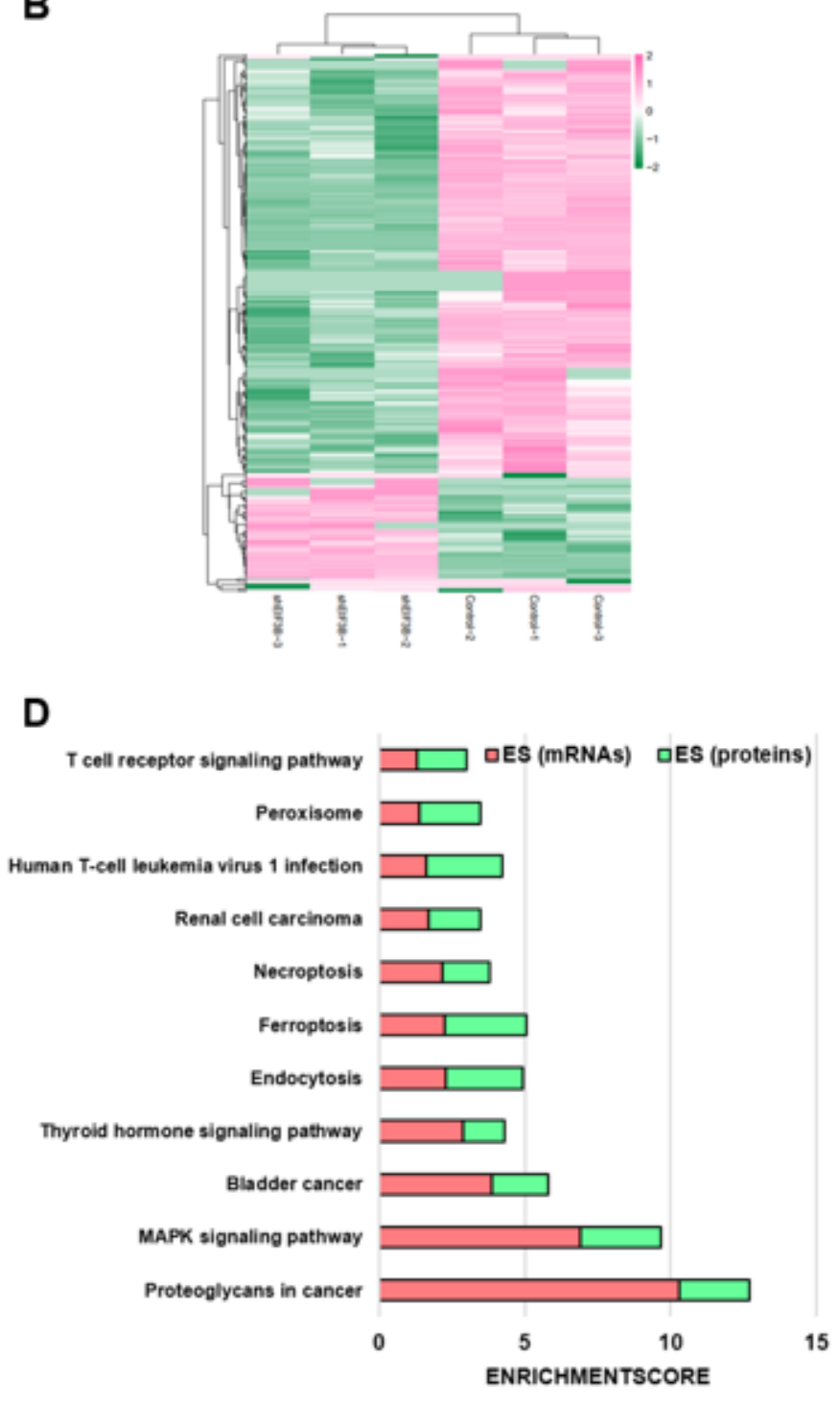

Figure 3 
Integrated transcriptomic and proteome analysis reveals potential pathways of EIF3B. (A) A total of 976 mRNAs were significantly deregulated after EIF3B was knockdown, and their expression was shown with heatmap; (B) A total of 425 proteins were significantly deregulated after EIF3B was knockdown, and their expression was shown with heatmap; (C) In gene level, 14 genes showed both deregulation in mRNA and protein level, and 12 pathways were both significantly enriched; (D) The common 12 enriched pathways was shown, according to pathway enrichment score (ES=-log10 P-value).

\section{A}

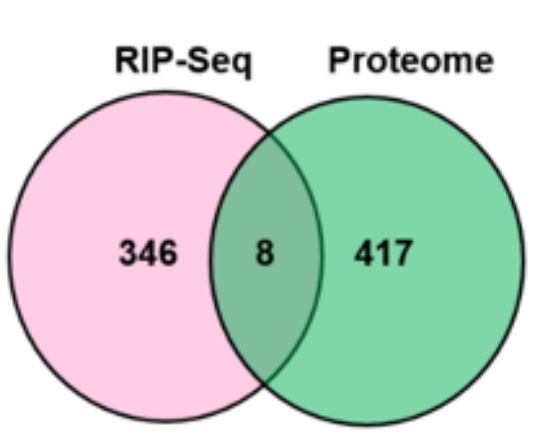

B

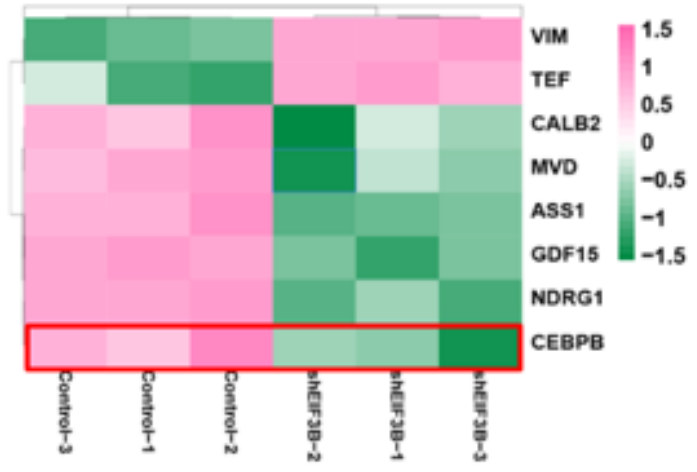

C

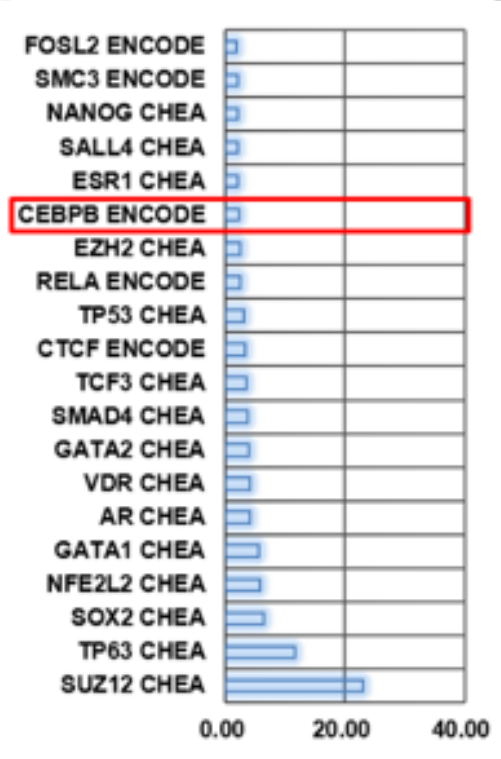

D

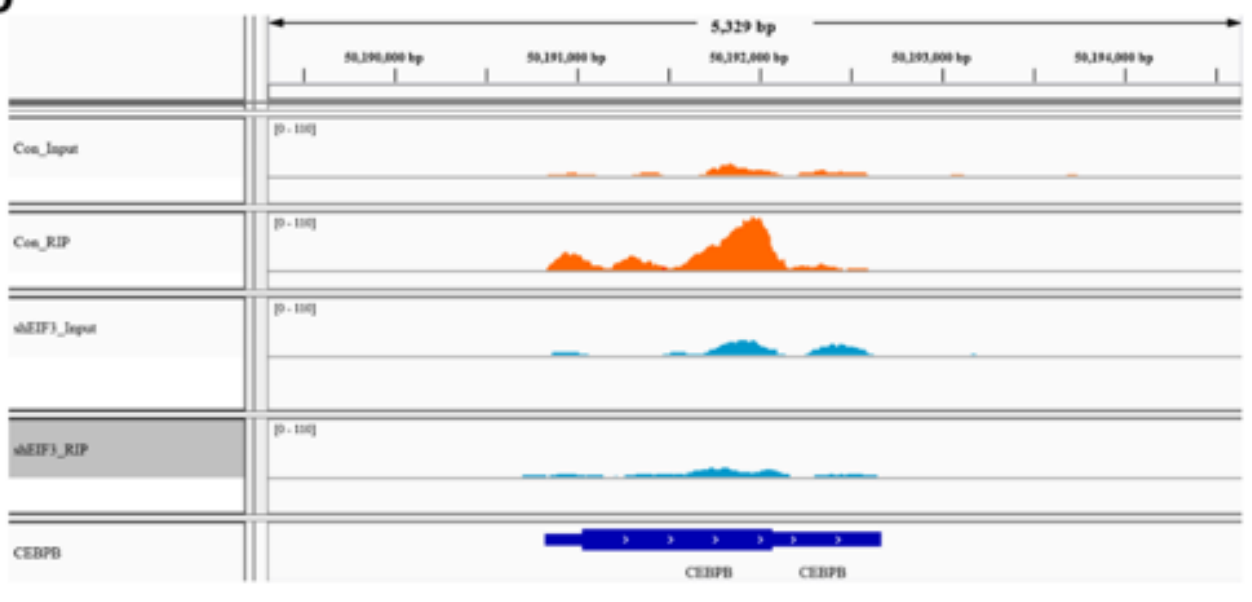

\section{Figure 4}

\section{RIP-Seq analysis reveals CEBPB as a direct target of EIF3B.}

(A)The RIP-Seq and proteome analysis uncovers 8 genes, with both significant differential expression in protein level, (B) and direct mRNA binding affinity by EIF3B; (C) The significantly deregulated mRNAs was applied for ENCODE and ChEA Consensus Transcription factors prediction, and top20 transcription factors were shown; Based on the results of RIP-Seq, proteome and RNA-seq, transcription factor CEBPB 
was proposed to be a direct target of EIF3B. The Binding motif and peaks of EIF3B with CEBPB mRNA was shown in (D).

A
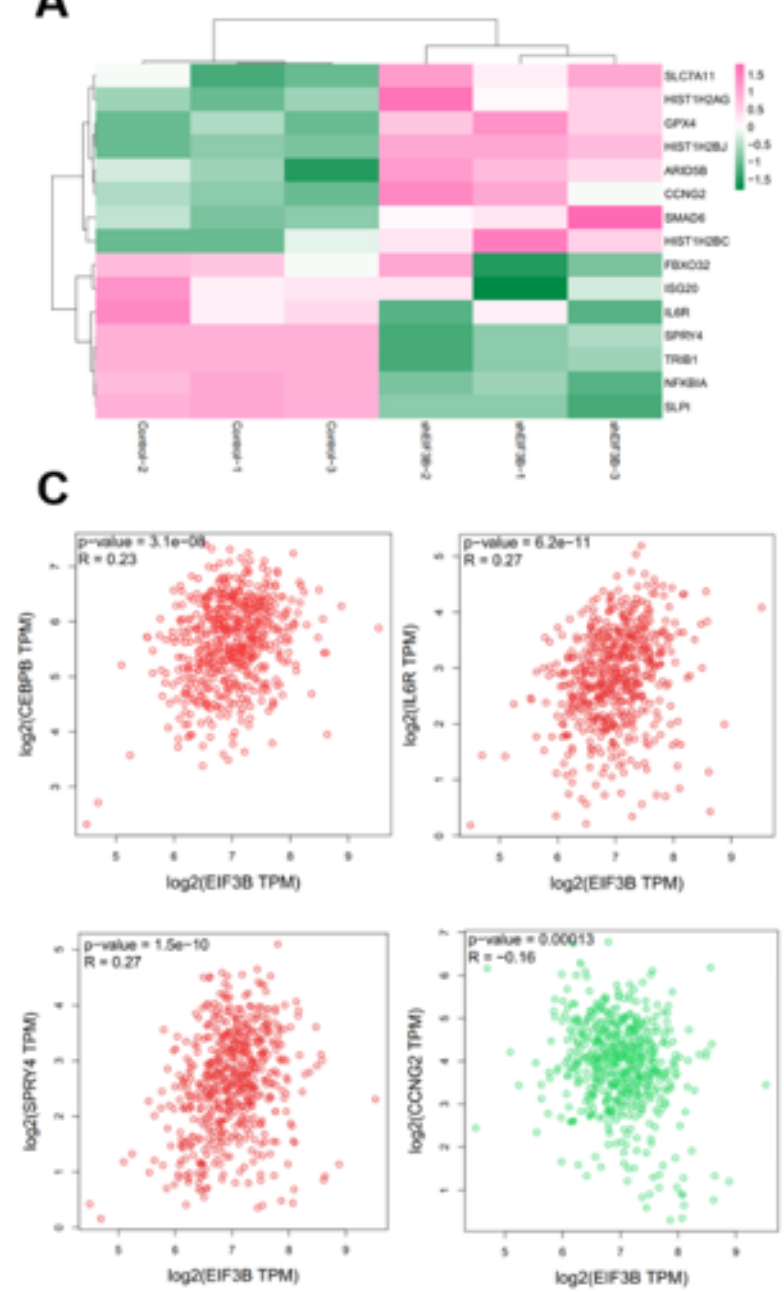

B

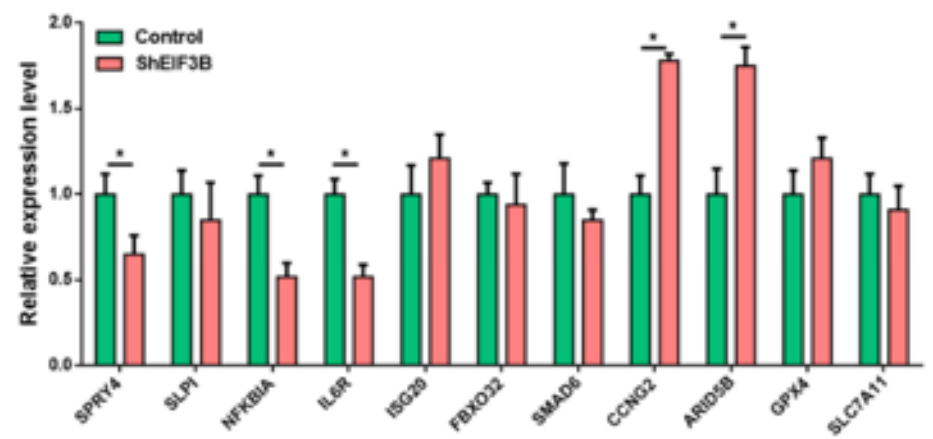

D

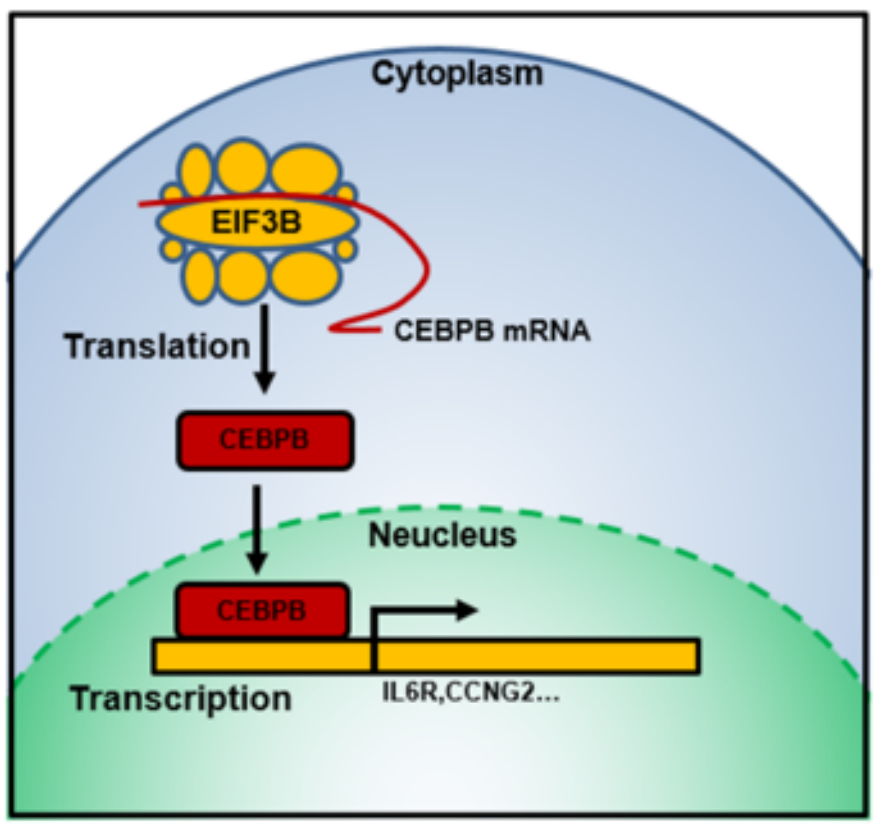

Figure 5

EIF3B regulates CEBPB targets expression and its role in HNSCC.

(A) The expression of CEBPB targets in control and EIF3B knockdown groups (shEIF3B) was shown with heatmap; (B) qRT-PCR was applied to validate the expression of CEBPB targets, and SPRY4, NFKBIA, IL6R and CCNG2 showed consistent trend with RNA-seq; (C) The co-expression of EIF3B and CEBPB targets were validated with TCGA HNSC dataset, and EIF3B showed significant positive correlation with CEBPB, SLPI, IL6R, SPRY4; and negative correlation with CCNG2. (D) EIF3B promotes HNSC progression by directly binding and promoting CEBPB translation, and activating downstream targets (e.g. IL6R, CCNG2) transcription and expression.

\section{Supplementary Files}


This is a list of supplementary files associated with this preprint. Click to download.

- GraphicAbstract.png 